\title{
Parallel calculations in the construction of the kinetic model of benzylidene benzylamine synthesis
}

\author{
I.V. Akhmetov ${ }^{1}$, I.M. Gubaydullin ${ }^{1,2}$ \\ ${ }^{1}$ Ufa State Technological Petroleum University, Kosmonavtov street 1, 450062, Ufa, Russia \\ ${ }^{2}$ Institute of Petrochemistry and Catalysis Russian Science Academy, Prospect Oktyabrya 141, 450075, Ufa, Russia
}

\begin{abstract}
In this paper, a kinetic model of the benzylidene benzylamine synthesis reaction has been developed. The optimal rate constants for the stages and activation energies are found. When searching for kinetic parameters, the OpenMP parallel computing technology was used. An effective number of flows are determined, in which the solution of inverse problems is most effective.
\end{abstract}

Keywords: kinetic model; rate constants; inverse kinetic problem; parallel calculations; OpenMP

\section{Introduction}

The catalytic reaction of the synthesis of the N-benzylidene benzylamine aromatic compond has a wide range of applications. $\mathrm{N}$-benzylidene benzylamine is known as an indicator for the quantitative determination of organolithium compounds by the titrimetric method and is the starting compound for the synthesis of a number of heterocycles $[1,2]$.

To study the mechanism of the synthesis reaction, it is necessary to construct a kinetic model, the solution of the inverse kinetic problems for which is complicated, because it is the most difficult and time consuming stage of kinetic model development.

Usage of parallel calculations is becoming more and more popular as a method of mathematical processing of experimental data because of the increasing complexity of obtained detailed information on chemical reactions.

The inverse problems of chemical kinetics refer to such physico-chemical problems that involve a significant amount of computations [3]. High-performance computing systems usage fundamentally changed the possibilities of complex chemical processes analysis: a detailed analysis of complex kinetic models with a large amount of experimental information has became available; The time for kinetic models construction has been reduced in many times; The accuracy of decisions has increased.

At present, solutions of inverse kinetic problems are proposed with the use of parallel calculations on cluster systems and graphics processors. Computer systems with multicore processors are actively introduced, the advantages of which are availability and ease of use, which expands the possibilities of their application in scientific researches $[4,5]$.

In this paper we propose a method for kinetic parameters searching using parallel calculations technology on multi-core systems for kinetic models construction of chemical reactions on metal complex catalysis with the aim of studying time reducing and mastering new catalytic processes.

\section{Kinetic model}

To understand the physical and chemical nature of the catalytic reaction, the subsequent mathematical modeling of the catalytic process and the definition of the conditions for its industrial realization, it is necessary first of all to develop its kinetic and mathematical models [6].

The fundamental basis for catalytic processes modeling is, first of all, the detailed studies of the physical and chemical nature of chemical reactions, since the quantitative characteristics obtained in the practical experiment and refined in numerical experiments will allow to develop kinetic models that will become a reliable basis for subsequent research.

The kinetic model of the process is a set of elementary stages, reactions and equations characterizing the dependence of the rate of chemical transformation on the reaction parameters: pressure, temperature, reagent concentrations, etc. Such dependencies are determined on the basis of experimental data obtained in the practical experiment while changing reaction parameters at the range of industrial conditions. The model developed in this way is the first level of the catalytic reactor model and the basis for later solving static and dynamic problems which arise in the development of technological processes.

The development of kinetic models, which is given in this paper, is based on experimental data of the benzylidene benzylamine synthesis which was obtained in the laboratory of hydrocarbon chemistry in IPC RAS. During the experiments, a new original method to include carboxyl group in pyrrols compounds, based on interaction between pyrrols and $\mathrm{CCl}_{4} \mathrm{CH}_{3} \mathrm{OH}-$ catalyst system.

Based on the analysis of the experimental data and the results of tit mathematical processing, the following scheme of chemical transformations and the corresponding kinetic equations (1)-(2) are proposed:
1. $\mathrm{C}_{1}+\mathrm{C}_{2} \rightarrow \mathrm{C}_{3}+\mathrm{C}_{4}$
$\mathrm{W}_{1}=\mathrm{k}_{1} \mathrm{C}_{1} \mathrm{C}_{2}$
2. $\mathrm{C}_{3} \rightarrow \mathrm{C}_{5}+\mathrm{C}_{6}$
3. $\mathrm{C}_{5}+\mathrm{C}_{1} \rightarrow \mathrm{C}_{7}+\mathrm{C}_{8}$
$\mathrm{W}_{2}=\mathrm{k}_{2} \mathrm{C}_{3}$
4. $\mathrm{C}_{8}+\mathrm{C}_{6} \rightarrow \mathrm{C}_{9}$
$\mathrm{W}_{3}=\mathrm{k}_{3} \mathrm{C}_{5} \mathrm{C}_{1}$
$\mathrm{W}_{4}=\mathrm{k}_{4} \mathrm{C}_{8} \mathrm{C}_{6}$ 
$\mathrm{C}_{\mathrm{i}}$ - concentration of the components, mol/l: $\mathrm{C}_{1}=\mathrm{C}_{7} \mathrm{H}_{9} \mathrm{~N}$ - benzylamine, $\mathrm{C}_{2}=\mathrm{CCl}_{4}-$ carbon tetrachloride, $\mathrm{C}_{3}=\mathrm{C}_{7} \mathrm{H}_{8} \mathrm{NCl}-$ chlorobenzylamine, $\mathrm{C}_{4}=\mathrm{CHCl}_{3}$ - chloroform, $\mathrm{C}_{5}=\mathrm{C}_{7} \mathrm{H}_{7} \mathrm{~N}$ - 1- phenylmethaneimine, $\mathrm{C}_{6}=\mathrm{HCl}$ - hydrochloric acid, $\mathrm{C}_{7}=\mathrm{C}_{14} \mathrm{H}_{13} \mathrm{~N}$ benzilidenbenzilamin, $\mathrm{C}_{8}=\mathrm{NH}_{3}$ - ammonia, $\mathrm{C}_{9}=\mathrm{NH}_{4} \mathrm{Cl}$ - ammonium chloride; $\mathrm{k}_{\mathrm{j}}$ - kinetic rate constant of $\mathrm{j}$-th reaction, $1 \cdot \mathrm{mol}^{-}$ ${ }^{1} \cdot h^{-1}(j=1,3,4), h^{-1}(j=2), W_{j}-j$-th rate of reaction, $\mathrm{mol} /(1 \cdot h)$.

The kinetic equations of the transformation scheme (1) are analyzed in accordance to the law mass action. The correct description of the laboratory reactor with a stirrer is the ideal mixing model:

$$
\begin{aligned}
& \frac{d \bar{N}}{d t}=F_{N}, F_{N}=\frac{1}{V_{o}} \sum_{j=1}^{J} \delta_{j} \omega_{j}, \delta_{j}=\sum_{i=1}^{I} v_{i j} \\
& \frac{d X_{i}}{d t}=\frac{F_{i}-X_{i} F_{N}}{\bar{N}}
\end{aligned}
$$

with the initial conditions: $t=0, X_{i}=X_{i}^{o}, \bar{N}=1$, where $\overline{\mathrm{N}}=\mathrm{C} / \mathrm{C}_{\mathrm{o}}$ - relative change in the number of moles of the reaction mixture; $\mathrm{C} \mathrm{и} \mathrm{C}_{\mathrm{o}}$ - the molar density and its initial value, mol/l; $\mathrm{X}_{\mathrm{i}}=\mathrm{C}_{\mathrm{i}} / \mathrm{C}$ - concentration of the components, the mole fractions; $\mathrm{V}_{\mathrm{o}}$ - the volume of the reaction space, $1 ; \omega_{\mathrm{j}}=\mathrm{W}_{\mathrm{j}} / \mathrm{C}_{\mathrm{o}}-$ given reaction rate, $\mathrm{h}^{-1} ; \mathrm{J}$ - number of stages of chemical transformation; $\mathrm{I}-$ the number of components.

The right sides of the systems of equations (2)-(3) have the following form:

$$
F_{1}=-\omega_{1}-\omega_{3} ; F_{2}=-\omega_{1} ; F_{3}=\omega_{1}-\omega_{2} ; F_{4}=\omega_{1} ; F_{5}=\omega_{2}-\omega_{3} ; F_{6}=\omega_{2}-\omega_{4} ; F_{7}=\omega_{1} ; F_{8}=\omega_{3}-\omega_{4} ; F_{9}=\omega_{4} ; F_{n}=\omega_{2}-\omega_{4}
$$

\section{Usage of parallel calculations}

For the parallel solution of the inverse kinetic problem the most effective method is genetic algorithm which is based on the idea of breeding, borrowed from biology, that is, the preferential multiplication of the fittest individuals [7]. The practical application of the genetic algorithm in all known cases led to positive results [8]. It is shown that the genetic algorithm, unlike the gradient methods of minimization, is a universal method for searching for an optimum regardless of the complexity of the functions [8]. The sequence of operations that form the basis of the genetic is described below.

At the first step of the algorithm, an initial population is created randomly, consisting of $\mathrm{N}$ individuals ( $\mathrm{N}$ points in the space of kinetic parameters, each point has $\mathrm{m}$ coordinates - parameter values). At the stage of mutation, the individuals change in accordance with a predetermined mutation operation, in which the coordinate/parabolic descent from the points of space was taken. At the stage of selection, a certain proportion of the whole population is selected, which will remain "alive" at this stage of evolution. The probability of survival of an individual depends on the value of the fittest function for this individual; as a function of the fittest $\mathrm{s}$ is the residual functional. The proportion of surviving $\mathrm{s}$ is a parameter of the genetic algorithm, and according to the results of selection from $\mathrm{N}$ individuals of the population, the total population will include $\mathrm{sN}$ individuals. In the case under consideration, $s=1 / 2$. When forming a new generation, a crossing is used - to produce a descendant, two parents are needed. To form a new point in the parameter space, one point from the "survivors" and one of the "dying" are selected as parents, and the crossing is done by choosing $\mathrm{m} / 2$ coordinates from the first point and the remaining ones from the second point; while the descendant inherits the traits of both parents. Specimens for reproduction are selected from the entire population, and not from surviving elements at the first step in order to exclude the possibility of population degradation. This set of actions is repeated iteratively, so the "evolutionary process", which lasts for several life cycles (generations), is modeled until the criterion for stopping the algorithm is fulfilled, which is any of the conditions:

1) finding a global or suboptimal solution;

2) the exhaustion of the number of generations released for evolution;

3) the exhaustion of the time allowed for evolution.

Parallelization of the calculation process takes place at the stage of initial filling, when the given pseudo-random points in the parameter space are uniformly distributed over the flows. Each mutation is mutated independently; The data is exchanged at the selection stage. At the same time, the autonomous work time of processes significantly exceeds the time of internuclear interactions, which determines the effectiveness of this algorithm (Fig. 1).

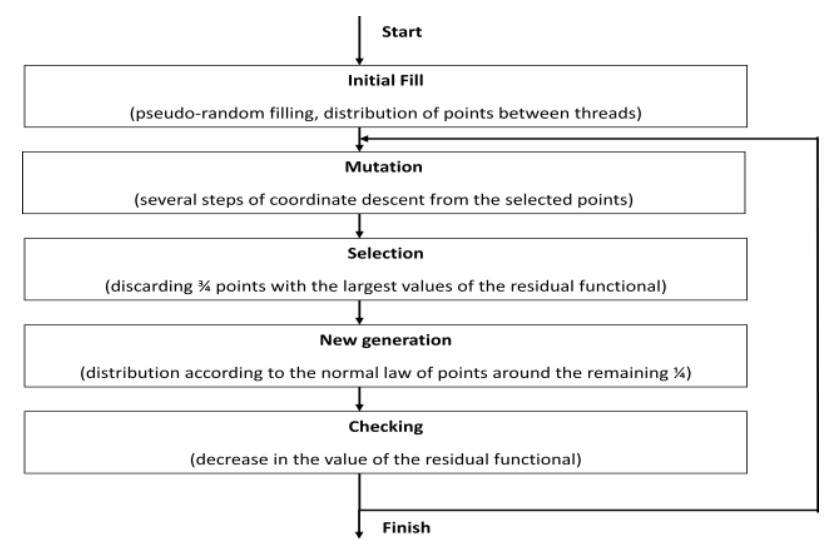

Fig. 1. Genetic algorithm. 


\section{Results of computational experiments}

The numerical values of the found rate constants of the stages and activation energies for the synthesis of benzylidene benzylamine are presented in Table 1.

Table 1. Kinetic parameters for synthesis of benzilidenbenzilamina.

\begin{tabular}{ccc}
\multicolumn{2}{c}{ Kinetic constants at $23^{\circ} \mathrm{C}, \mathrm{h}^{-1}$} & $\mathrm{E}_{\mathrm{i}}, \mathrm{kcal} / \mathrm{mol}$ \\
\hline $\mathrm{k}_{1}$ & $1.5 \times 10^{-2}$ & 10.6 \\
\hline $\mathrm{k}_{2}$ & 4.7 & 7.7 \\
\hline $\mathrm{k}_{3}$ & 13.4 & 1.6 \\
\hline $\mathrm{k}_{4}$ & 0.6 & 0.4
\end{tabular}

Estimating the efficiency of parallelization when testing a program on a computational cluster showed us that the parallel program works efficiently with increasing number of threads. When solving the inverse kinetic problem for the benzylidene benzylamine synthesis reaction from all experimental data, the total calculation time was 60 hours at 1 core, 3.5 hours using 18 fluxes (Fig. 2 and Fig. 3).

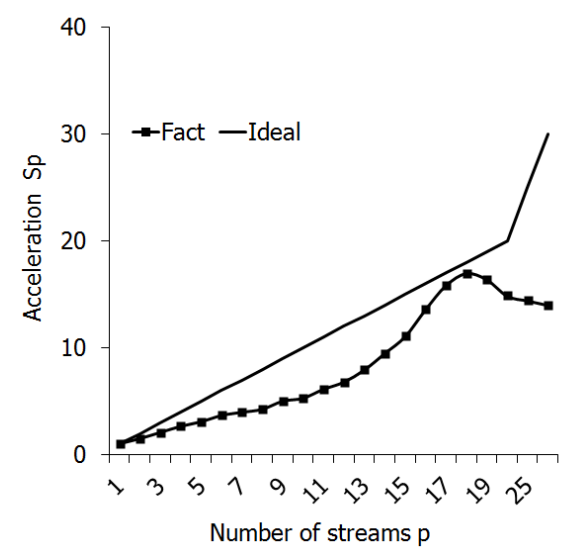

Fig. 2. Efficiency.

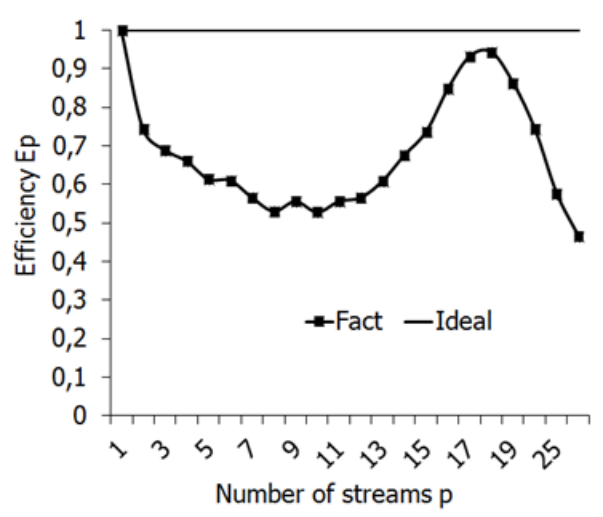

Fig. 3. Accelerate.

The adequacy of the constructed model with usage of parallel calculation of the display by comparing the calculated and experimental data on the yield of the desired product, benzylidene benzylamine (Fig. 4).

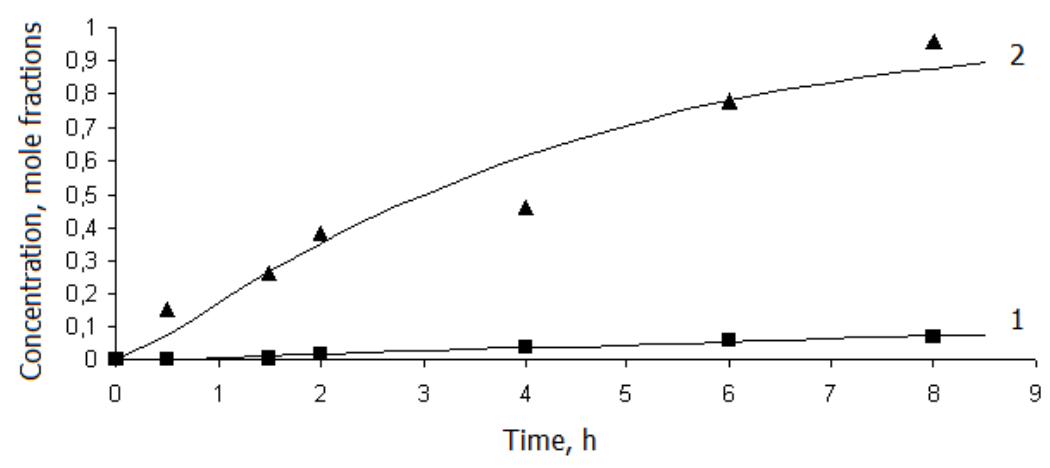

Fig. 4. Comparison of the calculated and experimental temperatures of $85 C^{0}(2)$ and $23 C^{0}(1)$. 


\section{Conclusion}

Thus, an algorithm has been developed for using multi-core computing systems to solve the inverse problems of chemical kinetics. The method is implemented as a software package that includes a database of kinetic studies, sequential and parallel algorithms for solving systems of ordinary differential equations, implemented on single-core and multi-core computing systems.

The informational and analytical systems have been developed, the successful application of which in the development of kinetic models of reactions of aromatic and heterocyclic compounds synthesis of has shown the universality of the proposed system approach to solving inverse kinetic problems.

The system allows users to easily adapt to the development of kinetic models of various reactions due to the formation of new blocks in the database of experimental data, the selection or addition of new methods of data processing, the construction of mathematical models of the objects of varying complexity.

Based on the developed approach, a kinetic model for the benzylidene benzylamine synthesis reaction was constructed. It is shown that with the use of the information system, the computing process can be accelerated approximately in 18 times.

\section{References}

[1] Khusnutdinov RI, Bayguzin AR, Aminov RI. Synthesis of N-benzylamine benzilidenbenzilamina under the action of iron catalysts in CCl 4. Journal of Organic Chemistry 2012; 48 (8): 1063- 1065.

[2] Khusnutdinov RI, Baiguzina AR, Mukminov RR, Akhmetov IV, Gubaidullin IM, Spivak SI, Dzhemilev UM. New synthesis of pyrrole-2-carboxylic and pyrrole-2,5-dicarboxylic acid esters in the presence of iron-containing catalysts. Russian Journal of Organic Chemistry 2010; 46(7): 1053-1059.

[3] Akhmetov IV, Gubaydullin IM. Analysis of methods for solving inverse problems of chemical kinetics with the use of parallel computing. PCT 2016 Proceedings of the 10th Annual International Scientific Conference on Parallel Computing Technologies.CEUR Workshop Proceedings 2016; $402-410$.

[4] Akhmetov IV. Multinuclearity in inverse kinetic problems. Scientific service in the Internet: search for new solutions Proceedings of the International Supercomputer Conference 2012; 656- 661.

[5] Akhmetov IV. Development of kinetic models for reactions of synthesis of aromatic and heterocyclic compounds based on multicore computing systems. Parallel Computing Technologies 2013. Proceedings of the International Scientific Conference 2013; 268- 277.

[6] Slinko MG. Modeling of chemical reactors. Novosibirsk: Science, 1968; 96 p.

[7] Nikitin AV, Nikitina LI. Evolutionary model of optimization of modular associative memory for data flow machines based on genetic algorithm. Programming 2002; 6: 31- 42 .

[8] Chernyshev O, Borisov A. Comparative analysis of solving optimization problems by genetic and gradient methods. Transport and Telecommunication 2007; 8(1): 40-52. 